\title{
Variación actancial en quichua santiagueño: los aplicativos (parte I)
}

\section{Actantial Variation in Santiago del Estero Quichua: the Applicatives (Part I)}

\author{
Lelia Inés Albarracín \\ Universidad Nacional de Santiago del Estero \\ ORCID: 0000-0002-3904-6108 \\ leliainesalbarracin@gmail.com
}

\author{
Regina Fernanda Gómez Nazar \\ Asociación de Investigadores en Lengua \\ Quechua (ADILQ) \\ ORCID: 0000-0001-9820-5060 \\ nazarfernanda77@gmail.com
}

\begin{abstract}
Resumen
En la variedad dialectal quechua hablada en la provincia de Santiago del Estero, República de Argentina, hay básicamente dos mecanismos que permiten incrementar la valencia verbal: a través de causativos o de aplicativos. Si bien se cuenta con una exhaustiva descripción de los mecanismos de incremento de valencia verbal mediante causativos y también una extensa explicación al funcionamiento de los denominados «sufijos transicionales», no hay para este último grupo de sufijos mayores detalles respecto a su utilización, con determinados verbos, en estructuras aplicativas y sin describir dicha operatoria como un mecanismo de ajuste valencial. A partir de investigaciones recientes y aún en curso, estamos realizando un replanteo de estos mecanismos, reanalizando a sufijos ya descriptos, pero ahora desde esta nueva perspectiva. En este trabajo, presentamos un avance de los resultados obtenidos hasta el presente.
\end{abstract}

Palabras clave: quechua, quichua, aplicativos, valencia verbal

\begin{abstract}
In the dialectal variety of Quechua spoken in the province of Santiago del Estero, Argentina, there are basically two mechanisms that allow the increase of verbal valence: through causatives or applicatives. Although there is an exhaustive description of the mechanisms of verbal valency increase by means of causatives and also an extensive explanation of the functioning of the so-called «transitional suffixes», there are no further details regarding their use with certain verbs in applicative structures and without describing this operation as a mechanism of valential adjustment. Based on recent and still ongoing research, we are rethinking these mechanisms, reanalyzing suffixes already described but now from this new perspective. In this paper, we present an advance of the results obtained so far.
\end{abstract}

Keywords: quechua, quichua, applicatives, verbal valency

Recibido: 14-02-2021

Aprobado: 20-02-2021

Publicado: 15-07-2021 
Variación actancial en quichua santiagueño: los aplicativos (parte I)

Lelia Inés Albarracín y Regina Fernanda Gómez Nazar

\section{Introducción}

En la variedad dialectal quechua conocida como «la quichua» $\mathrm{o}$ «quichua santiagueño» (hablada en la provincia de Santiago del Estero, Rep.Argentina), hay básicamente dos mecanismos que permiten incrementar la valencia verbal: a través de causativos o a través de aplicativos. En el caso de los causativos, son sufijos que han sido descriptos inicialmente por Alderetes (2001) y Nardi (2002), por medio de ejemplos de uso, sin que se haga mención a su función como tales. Recién en Albarracín de Alderetes (2011), hay una exhaustiva descripción de los mecanismos de ajuste de valencia verbal mediante causativos, como así también de las diátesis regresiva y progresivo-regresiva. La misma autora dedica también una extensa explicación al funcionamiento de los denominados «sufijos transicionales» o "sufijos de persona objeto» pero sin mencionar su utilización, con determinados verbos, en estructuras aplicativas y sin describir dicha operatoria como un mecanismo de ajuste valencial. En los trabajos de lingüística relacionados con el quichua santiagueño, salvo las contribuciones de Neil Myler (2016, 2018) sobre el aplicativo - $p u$, conocido como «benefactivo», no hay referencias concretas a los aplicativos. A partir de la obtención de nuevos registros y, fundamentalmente, gracias a la interacción con hablantes bilingües durante su proceso de formación universitaria, se están realizando investigaciones tendientes a hacer un replanteo crítico de estos mecanismos. En este trabajo, abordaremos dicha cuestión, reanalizando a sufijos ya descriptos pero ahora desde esta nueva perspectiva, y presentaremos un avance de los resultados obtenidos hasta el presente. Nuestro agradecimiento a Carlos Sosa More, Rosana Aguirre y Claudio Sebastián Basualdo, jóvenes hablantes maternos, por su inestimable colaboración.

\section{Los aplicativos}

Desde 1981, fecha en que Alec Marantz publicó su propuesta acerca de la categoría de aplicativo, y hasta el presente, son numerosos los autores que han realizado contribuciones sobre la temática de las construcciones aplicativas, aportando nuevas miradas, precisiones, restricciones y extensiones no siempre coincidentes. En la búsqueda de un marco teórico adecuado para nuestras investigaciones, nos han resultado particularmente útiles los trabajos sobre lenguas indígenas de nuestro país, la República Argentina, publicados por Carrió (2013, 2015), González

14 Lengua y Sociedad. Revista de Lingüística Teórica y Aplicada 
(2016), Fernández Garay (2012) y Censabella (2011) ${ }^{1}$, entre otros. Si bien los mismos no están relacionados con la lengua quichua de Santiago del Estero, tema central de este artículo, sí nos orientaron y facilitaron la búsqueda, entre tanto material bibliográfico disponible, de aquel que resultara pertinente para nuestras propias necesidades. Cabe señalar que los únicos trabajos sobre aplicativos relacionados con la lengua quichua, a los que pudimos tener acceso, son parte de la investigación llevada a cabo por Neil Myler $(2016,2018)$ sobre estructuras posesivas y que generosamente su autor puso a nuestra disposición.

Según Carrió (2013, p. 42), Marantz concibe a los aplicativos como afijos con estructura argumental independiente que provocan cambios en la función gramatical y que conllevan consecuencias morfológicas significativas, se trataría de operaciones no léxicas sino sintácticas con repercusión morfológica. Además, siempre siguiendo a Carrió, según Marantz (1981, p. 259) estos afijos portan su propia estructura argumental, la cual se ensambla a las raíces que los hospedan. Carrió (2013) señala además:

Así, estos verbos, mediante una operación sintáctica denominada applicative, incrementan su estructura argumental (o, en otros términos, aumentan su valencia) dado que introducen un participante periférico a la estructura argumental básica del verbo, a la vez que reducen el número de constituyentes superficiales. Esta operación permite que la información que en algunas lenguas se presenta como adjunto (a nivel sintáctico) sea, para estos casos, un complemento regido por el afijo que está ensamblado en el núcleo verbal y que reciba rol temático en una posición argumental. En estos casos, el «nuevo complemento» se presenta como un «objeto aplicado» sobre el que tiene alcance el aplicativo sufijado a la base verbal. (p. 42)

Para nuestra investigación, hemos prestado particular atención a aquellos estudios en donde las construcciones aplicativas son descritas prototípicamente como transitivizantes, puesto que involucran una base verbal intransitiva la cual deviene transitiva, es decir, un verbo que exige la presencia de un objeto directo. Tal como lo señala Payne (1997):

Algunas lenguas tienen operaciones por las que un verbo es marcado para el rol semántico de un objeto directo. Aquí nos referiremos a tales

1 Particularmente agradecemos a Marisa Censabella quien, hace ya algún tiempo, nos acercó generosamente material sobre la temática. 
Variación actancial en quichua santiagueño: los aplicativos (parte I)

Lelia Inés Albarracín y Regina Fernanda Gómez Nazar

operaciones como aplicativos, aunque también se les denomina «avances» o «promociones» a objeto directo. En la mayoría de los casos, un aplicativo puede describirse claramente como una operación de aumento de valencia que trae a un participante periférico al centro del escenario convirtiéndolo en un objeto directo. El «nuevo» objeto directo se denomina a veces objeto aplicado. En el caso de los verbos que ya tienen un objeto directo, el aplicativo da lugar a un verbo de tres argumentos (ditransitivo), o el objeto directo «original» deja de expresarse. En este último caso, el aplicativo no puede considerarse como un dispositivo de incremento de valencia, ya que el verbo original y el verbo resultante tienen el mismo número de argumentos; más bien, el aplicativo se limita a atribuir un nuevo rol semántico, antes periférico, al objeto directo. (pp. 186-187)

Por su parte, Peterson (2007, p. 39) define a las construcciones aplicativas, en términos de su morfosintaxis, como estructuras oracionales en las cuales un participante periférico u oblicuo es promovido a argumento central (usualmente objeto directo) y las mismas llevan una marca morfológica expresa que se manifiesta en el sintagma verbal. Si bien Peterson señala que la construcción aplicativa suele ser altamente productiva en una parte significativa del léxico verbal, no es el caso de la lengua quichua ya que, como veremos, se aplica de manera restrictiva a determinado tipos de verbos.

Siempre de acuerdo con Peterson (2007, p. 40), si una lengua tiene una construcción que podría caracterizarse como aplicativa, el rol semántico más común del objeto aplicado es el de recipiente y/o benefactivo/malefactivo. Además, este autor precisa que hay lenguas en las que la función del objeto aplicado no se limita al rol de destinatario o benefactivo/malefactivo, sino que también pueden presentar múltiples aplicativos con distintos roles semánticos (causales, instrumentales, comitativos, etc.).

\section{Los aplicativos en la quichua de Santiago}

La lengua quichua presenta dos tipos de derivados para aumentar la valencia verbal, los causativos, ya descriptos y analizados por Albarracín de Alderetes (2011), y los aplicativos. Y aquí resulta pertinente recordar la afirmación de Martin (2000, p. 377) en cuanto a que la diferencia entre ambos es clara: el causativo manipula el punto inicial del evento, es decir que agrega un agente a los ya existentes, en tanto que el aplicativo, manipula el punto final, ya que agrega un objeto.

16 Lengua y Sociedad. Revista de Lingüística Teórica y Aplicada 
El propósito de este trabajo es describir y analizar tres sufijos de la lengua quichua de Santiago del Estero, dos de los cuales pueden cumplir la función de aplicativos (además de sus funciones habituales como marcadores de persona objeto) y de un aplicativo alto (Pylkkänen, 2008, p. 13) ${ }^{2}$ como lo es el benefactivo - $p u$.

Se trata de los sufijos de interacción -su 'marcador de 2. ${ }^{a}$ persona objeto', -wa - $a$ 'marcador de 1. ${ }^{\text {a }}$ persona objeto' y el benefactivo/malefactivo - $p u$. Los dos primeros habitualmente se utilizan con verbos transitivos en el sistema de conjugación históricamente denominado «transiciones» (relaciones sujeto-objeto) o «conjugación transitiva», que se caracteriza porque cuando el objeto es un pronombre de 1. ${ }^{a}$ o de 2. ${ }^{a}$ persona, (es decir, los dos participantes directos de la comunicación), el objeto se incorpora de manera directa al verbo mediante un sufijo.

Por otro lado, y sin formar parte de ese paradigma de «conjugación transitiva», la función habitual de - $p u$ es indicar que la acción se realiza en provecho (benefactivo) o en perjuicio (malefactivo) de algún otro. Más adelante veremos que el argumento aplicado puede asociarse a una serie de interpretaciones, además de la benefactora.

Son tres los casos que analizaremos en el presente trabajo, con aplicativos operando sobre verbos intransitivos de movimiento, existenciales y sensoriales.

Cabe aquí advertir que no todos los verbos intransitivos admiten la incorporación directa de estos sufijos, tal es el caso, por ejemplo, del verbo rimay 'hablar' que solo los acepta si previamente ha sido transitivizado mediante un causativo $y$, en tal caso, los aplicativos solo se limitan a identificar a la persona objeto. Es decir, hay situaciones en las que el aplicativo no se comporta como un dispositivo de incremento de valencia sino que produce una reespecificación o reacomodamiento de la estructura argumental.

\section{Aplicativos con verbos de movimiento}

De acuerdo con Fernández Garay (2012, p. 4), hay dos tipos de oraciones universales, las intransitivas y las transitivas. Las intransitivas presentan un solo argumento central que llamaremos $S$ (sujeto de intransitiva), en tanto que las transitivas poseen dos argumentos centrales, que llamaremos A (agente) y P (paciente). $\mathrm{A}$ es el que inicia o controla la actividad y $\mathrm{P}$ es el que se halla afectado por dicha

2 Pylkkänen propone dos tipos de aplicativos: los aplicativos altos, que denotan una relación entre un evento y un individuo, y los aplicativos bajos, que denotan una relación entre dos individuos. 
Variación actancial en quichua santiagueño: los aplicativos (parte I)

Lelia Inés Albarracín y Regina Fernanda Gómez Nazar

actividad. Formando parte de los argumentos centrales encontramos a veces un tercer argumento llamado R (recipiente o beneficiario) que ocurre en oraciones ditransitivas.

Los sufijos -su y - wa $\sim-a$, cuando se utilizan con algunos verbos intransitivos típicos de movimiento o que implican algún tipo de movimiento, de acuerdo con nuestra propuesta, funcionan como aplicativos porque aumentan la valencia a los efectos de incorporar un actante en función de objeto directo. Por su parte, Myler (2018) ofrece una explicación diferente, postulando un morfema aplicativo nulo $\emptyset$, responsable del incremento valencial, a la par que mantiene la condición de marcadores de objeto para -su y -wa.

Por ejemplo, el verbo lloqsiy 'salir', en una construcción intransitiva tiene un solo argumento central en función de sujeto:

$$
\begin{aligned}
& \text { pay lloqsi-n } \\
& \text { él/ella salir-3 } \\
& \mathrm{S} \\
& \text { 'él/ella sale' }
\end{aligned}
$$

Al incorporar en la estructura del verbo al aplicativo $-w a \sim-a$, la valencia se incrementa para dar lugar a un verbo transitivo y trae a un participante periférico (en este caso siempre de $1 .{ }^{\text {a }}$ persona) al centro del escenario convirtiéndolo en un objeto directo:

$\begin{array}{llll}\text { chunka-y-pi } & \text { suk } & \text { qotu } & \text { lloqsi-a- } n \\ \text { pierna-IPOSS-LOC } & \text { una } & \text { protuberancia } & \text { salir-APPL-3 } \\ & & \text { S } & \text { OD }\end{array}$

'me salió una protuberancia en la pierna'

Si el objeto aplicado estuviera explícito fuera de la conformación morfológica del verbo, por ejemplo mediante un pronombre personal (noqa en este caso), tendría que estar marcado con el acusativo - $t a$, siendo su rol semántico el de Paciente:

$\begin{array}{lllll}\text { chunka-y-pi } & \text { suk } & \text { qotu } & \text { noqa-ta } & \text { lloqsi-a-n } \\ \text { pierna-IPOSS-LOC } & \text { una } & \text { protuberancia } & \text { yo-ACC } & \text { salir-APPL-3 } \\ & & \text { S } & \text { OD } & \end{array}$

'(a mí) me salió una protuberancia en la pierna'

18 Lengua y Sociedad. Revista de Lingüística Teórica y Aplicada 
Veamos otro ejemplo, esta vez con el verbo sutuy cuyo significado nuclear es 'gotear'; en una construcción intransitiva tiene un solo argumento central en función de sujeto:

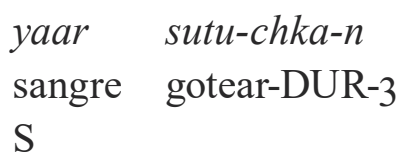

Al incorporar en la estructura del verbo al aplicativo -su, la valencia se incrementa para traer a un participante periférico (en este caso siempre de $2 .{ }^{a}$ persona) al centro del escenario convirtiéndolo en un objeto directo:

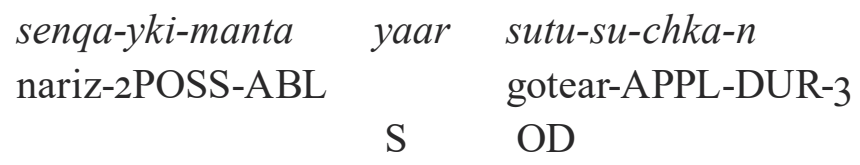

'te sale sangre de la nariz'

Si el objeto aplicado estuviera explícito fuera de la conformación morfológica del verbo, por ejemplo mediante un pronombre personal (qam en este caso), tendría que estar marcado con el acusativo - $t a$, siendo su rol semántico el de Paciente:

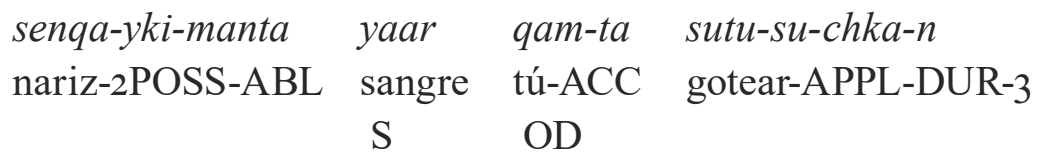

'(a ti) te sale sangre de la nariz'

Para Myler, el ejemplo anterior debería analizarse así, incorporando un morfema aplicativo nulo:

sutu-Ø-su-chka-n

gotear-APPL-2OBJ-DUR-3

Los siguientes son otros dos ejemplos con verbos intransitivos de movimiento (recordemos que con -su el objeto aplicado es siempre de 2. ${ }^{a}$ persona): 


$$
\begin{array}{lll}
\text { ñawi-s-ni-yki-pi } & \text { allpa } & \text { yayku-so-nqa } \\
\text { ojo-PL-EST-2POSS-LOC } & \text { tierra } & \text { entrar-APPL-3FUT } \\
& \mathrm{S} & \text { OD }
\end{array}
$$

'te va a entrar tierra en los ojos'

Entre las fórmulas de cortesía de la lengua quichua es muy recurrida la siguiente expresión que, a pesar de ser utilizada también en otras variedades quechuas (por ejemplo, cf. Albó, 1977, p. 230), en nuestra opinión, es un calco de una oración desiderativa castellana que porta el sufijo exhortativo de $3 .{ }^{\text {a }}$ persona singular -chun. El objeto aplicado qam-ta de $2 .^{a}$ persona y señalado por -su, no está explicitado:

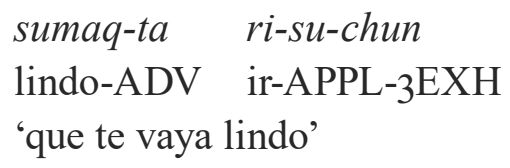

Por último, consideraremos el sufijo verbal - $p u$ 'benefactivo/malefactivo', el cual cuando es utilizado en un verbo transitivo se comporta, según Albarracín de Alderetes (2011, p. 244), como «un orientador actancial, que desvía la orientación de la acción hacia otra instancia (hacia el tercer actante) diferente al sujeto y al objeto del verbo, y permite indicar que la acción se realiza en provecho o en perjuicio de algún otro». Si el verbo es unitransitivo, - $p$ u se comporta como un aplicativo incrementando la valencia verbal, pero si el verbo es ditransitivo, $-p u$ se comporta como un benefactivo puro sin ajuste de valencia.

Este sufijo tiene la particularidad de que puede ser utilizado con un grupo reducido de verbos intransitivos, con el propósito de transitivizar al verbo e indicar que el objeto es de $3 \cdot{ }^{\text {a }}$ persona. Tal situación ocurre con algunos verbos intransitivos típicos de movimiento o que implican algún tipo de movimiento, con los cuales - $p$ u funciona como aplicativo aumentando la valencia, a los efectos de incorporar un actante en función de objeto de 3 a $^{\text {a }}$ persona. Por ejemplo:

$$
\begin{array}{ll}
\text { yaar mana } & \text { lloqsi-n } \\
\text { sangre no } & \text { salir-3 } \\
\text { 'no sale sangre (lit.: la sangre no sale)' }
\end{array}
$$

$\begin{array}{llll}\text { yaar } & \text { pay-ta } & \text { mana } & \text { lloqsi-pu- } n \\ \text { sangre } & \text { él/ella-ACC } & \text { no } & \text { salir-APPL-3 }\end{array}$

'no le sale sangre (lit.: a ella/él la sangre no le sale)' 
A continuación mostraremos que el rol semántico del argumento aplicado puede asociarse a una serie de interpretaciones, entre ellas la benefactora. En los siguientes ejemplos, con los verbos intransitivos ayqey 'huir', amuy 'venir' y riy 'ir', el sufijo - pu está utilizado en un contexto en donde la acción perjudica/ beneficia a una $3 .{ }^{\text {a }}$ persona:

$\begin{array}{lllll}\text { sumaq-lla-ta } & \text { ri-chka-nki } & \text { pero } & \text { ayqe-pu-y } & \text { avanza-su-pte-nqa } \\ \text { lindo-DLM-ADV } & \text { ir-DUR-2 } & \text { pero } & \text { huir-APPL- } & \text { avanzar-2OBJ- } \\ & & & \text { 2IMP } & \text { DS-3FUT }\end{array}$

'Estás yendo lindo, pero escapale cuando avanza sobre vos'

$\begin{array}{lll}\text { chuchu } \quad \text { amu-pu-sa } & k a-r a-\varnothing \\ \text { escalofrío } & \text { venir-APPL-PST.N } & \text { ser-PST-3 } \\ \text { 'Le había venido escalofrío' } & \end{array}$

Y mana pipas niyta atinman Andreataqa imaynachus ripora matrimoniunpé Nadie puede saber cómo le habrá ido a Andrea en su matrimonio

Pero en estos otros ejemplos, el aplicativo establece un punto de referencia a partir del cual se desvía la acción para entrar o salir del campo de visión (Itier, 1997, pp. 89-90), siempre en relación con una 3. ${ }^{a}$ persona, tal el caso de chayapuy 'llegarle' (acercándose a un punto de referencia) o anchupuy 'apartársele' (alejándose de un punto de referencia).

de poco a poquito anchu-po-ra-ni de poco a poquito alejarse-APPL-PST-I 'de poco a poquito me le alejé'

$\begin{array}{lllll}\text { puri-sa } & \text { ka-ra-Ø un mosito ancha } & \text { chaya-pu-s } \\ \text { andar-PST.N } & \text { ser-PST-3 un joven mucho } & \text { llegar-APPL-MS } \\ \text { 'había andado un mocito llegándole mucho' }\end{array}$

\section{Aplicativos con verbos sensoriales}

Cualquier proceso mental (sea de sensación, percepción, cognición o volición) implica dos participantes inherentes: un experimentador del proceso dotado 
Variación actancial en quichua santiagueño: los aplicativos (parte I)

Lelia Inés Albarracín y Regina Fernanda Gómez Nazar

de capacidad intelectiva y una representación del fenómeno experimentado. Entendemos por argumentos inherentes aquellos sin los cuales no se puede concebir el proceso designado.

En algunos verbos intransitivos sensoriales (o de percepción sensorial) es ineludible la utilización de los aplicativos -su y - wa $\sim-a$, para poder incrementar la valencia a los efectos de incorporar un actante en función de objeto.

Por ejemplo, al incorporar al aplicativo $-w a \sim-a$ en la estructura del verbo intransitivo, la valencia se incrementa para traer a un participante periférico (en este caso siempre de 1. ${ }^{a}$ persona, es decir, noqata 'a mí') al centro del escenario y sintácticamente, este argumento aplicado se comporta como 'objeto directo'.

Para ilustrar este caso, recurriremos al verbo de percepción gustay 'gustar', un préstamo del castellano que funciona únicamente como intransitivo:

$\begin{array}{lllll}\text { gusta-a- } q & \text { ka-ra- } \varnothing & \text { uyari-pu-y } & \text { yuya-pti-n } & \text { vida- } n \text {-ta } \\ \text { gustar-APPL- } & \text { ser-PST-3 } & \text { oir-APPL- } & \text { recordar-DS-3 } & \text { vida-3POSS- } \\ \text { PST.H } & & \text { INF } & & \text { ACC }\end{array}$

'(a mí) me gustaba escucharlo cuando recordaba su vida'

$\begin{array}{lllll}\text { mana } & \text { gusta-a-n-man-chu } & \text { cree-pu-ku-s ima } & \text { puri-na-yké } \\ \text { no } & \text { gustar-APPL-3- } & \text { creer-APPL- acaso } & \text { andar-NMLZ- } \\ & \text { COND-NEG } & \text { PL-MS } & & \text { 2-TOP }\end{array}$

'no me gustaría (a mí) que vos acaso andes creyéndoles'

La siguiente oración compleja es una subordinada complementaria de sujeto infinitiva, en donde la raíz subordinada danza- 'bailar' lleva el complementizador $-y$ y se encuentra en caso nominativo (eventualmente puede llevar el marcador de tópico -qa). Como señala Albarracín de Alderetes (2016, p. 153) este verbo subordinado puede tener un objeto directo, en este caso chamamés marcado con $-t a$. Mediante el aplicativo $-w a \sim-a$ se incrementa su valencia para incorporar un objeto aplicado de $1 .^{a}$ persona, expresado dentro de la propia morfología del verbo por dicho sufijo, y externamente por un pronombre personal correferente marcado con $-t a$.

$\begin{array}{llll}\text { noqa-ta } & \text { chamames-ta } & \text { gusta- } a-n & \text { danza-y-qa } \\ \text { yo-ACC } & \text { chamamés-ACC } & \text { gustar-APPL-3 } & \text { bailar-INF-TOP } \\ \text { 'me gusta bailar chamamés' } & & \end{array}$


Otros ejemplos (en el primero nótese que el acusativo - $t a$ aparece también en su forma apocopada $-t$ ):

noqa-t ancha gusta-a-n lokro-t miku-y yo-ACC mucho gustar-APPL-3 locro-ACC comer-INF 'a mí me gusta mucho comer locro'

chamames-ta gusta-a-n danza- $y$-qa

chamamés-ACC gustar-APPL-3 bailar-INF-TOP

'me gusta el bailar chamamés'

Respecto del aplicativo -su, la situación es idéntica que con $-w a \sim-a$ solo que el objeto aplicado es de $2 .{ }^{\text {a }}$ persona:

(qam-ta) gusta-su-n tanta-t ruwa-y

(tú-ACC) gustar-APPL-3 pan-ACC hacer-INF

'(a ti) te gusta hacer pan'

en donde -su hace referencia a una $2 .{ }^{\text {a }}$ persona representada fuera de la morfología del verbo por el pronombre correspondiente marcado con - ta. En estos tres últimos ejemplos, el verbo subordinado tiene un objeto directo también marcado con - $t a$. Es preciso señalar que en hablantes bilingües más jóvenes hemos registrado los mismos ejemplos pero con el verbo subordinado en caso acusativo ${ }^{3}$ :

${ }^{*}$ Noqa-t ancha gusta-a-n lokro-t miku-y-ta

${ }^{*}$ Qam-ta gusta-su-n tanta-t ruwa-y-ta.

Otro uso típico de estos aplicativos ocurre con ciertos verbos de sensación. En los siguientes ejemplos con los verbos intransitivos nanay 'doler' y chiriy 'hacer frío' podemos ver como - wa $\sim-a$ y -su incrementan la valencia verbal para traer a un participante periférico al centro del escenario convirtiéndolo en un objeto directo (de $1 .^{\mathrm{a}}$ y $2 .^{\mathrm{a}}$ persona respectivamente). El objeto aplicado puede estar representado externamente y de manera redundante por un pronombre personal correferente marcado con -ta.

3 Probablemente sea la consecuencia de una interferencia del castellano, en donde el verbo 'gustar' puede ser transitivo o intransitivo. 
Variación actancial en quichua santiagueño: los aplicativos (parte I)

Lelia Inés Albarracín y Regina Fernanda Gómez Nazar

$\begin{array}{ll}\begin{array}{l}\text { (noqa-ta) mana nana-a-n } \\ \text { yo-ACC no doler-APPL-3 }\end{array} & \begin{array}{l}\text { noqa-ta chiri-a-n } \\ \text { yo-ACC hacer frío-APPL-3 } \\ \text { '(a mí) no me duele' }\end{array} \\ \begin{array}{ll}\text { 'qam-ta) mana hana-su- } n & \text { qam-ta chiri-su- } n\end{array} \\ \begin{array}{l}\text { yo-ACC no } \\ \text { '(a ti) no te duele' }\end{array} & \begin{array}{l}\text { tú-ACC hacer frío-APPL-3 } \\ \text { 'te hace frío' }\end{array}\end{array}$

A continuación analizaremos el caso del aplicativo - $p u$ y para ello ejemplificaremos nuevamente con el verbo intransitivo de percepción gustay 'gustar'. El propósito del aplicativo es incrementar la valencia a los efectos de incorporar un actante en función de objeto de $3{ }^{\text {a }}$ persona. En la siguiente oración, el objeto aplicado es sipitas 'muchachita' y lleva la marca de objeto - $t a$.

Sipitastá cha tiyusimipa mishkenqa kaq kasa kara tukuymanta astaan gustapoqqa

'De todo, la miel de tiusimi era lo que a la muchachita más le gustaba'

Otros ejemplos con el intransitivo gustay:

$\begin{array}{llll}\text { (pay-ta) } & \text { danza-y-pas } & \text { gusta-po- } q & k a-r a-\varnothing \\ \text { él/ella-ACC } & \text { bailar-INF-TOO } & \text { gustar-APPL-PST.H } & \text { ser-PST-3 } \\ \text { '(a él) también le gustaba el bailar' } & \end{array}$

$\begin{array}{llll}(\text { pay-ta }) & \text { gusta-po-q } & k a-r a-\varnothing & \text { vinu } \\ \text { él/ella-ACC } & \text { gustar-APPL-PST.H } & \text { ser-PST-3 } & \text { vino } \\ \text { '(a él) le gustaba el vino' } & & \end{array}$

$\begin{array}{llll}\text { nataq } & \text { gusta-po-q } & k a-r a-\varnothing & l e e-y \\ \text { y también } & \text { gustar-APPL-PST.H } & \text { ser-PST-3 } & \text { leer-INF }\end{array}$

'y también (a él) le gustaba leer'

$\begin{array}{llllll}\text { farras-manta } & \text { astaan } & \text { gusta-po-q } & k a-r a-\varnothing & \text { naipes-pi } & \text { puklla-y } \\ \text { farras-ABL } & \text { más } & \text { gustar-APPL- } & \text { ser-PST-3 } & \text { naipes-LOC } & \text { jugar-INF } \\ & & \text { PST.H } & & & \end{array}$

'de las farras lo que más le gustaba (a él) era jugar a los naipes'

24 Lengua y Sociedad. Revista de Lingüística Teórica y Aplicada 
Cuando el objeto aplicado es plural o alude a un conjunto, el aplicativo - $p u$ lleva el pluralizador $-k u$, como en el siguiente ejemplo:

$$
\begin{array}{llll}
\text { gente- } t a & \text { ancha } & \text { gusta-pu-ko-ra-ø } & \text { música-nkuná } \\
\text { gente-ACC } & \text { mucho } & \text { gustar-APPL-PL-PST-3 } & \text { música-3POSS-TOP }
\end{array}
$$

'a la gente le gustó mucho su música'

Veamos ahora el caso de una oración compleja. En el siguiente ejemplo advertimos, por un lado, que en la subordinada complementaria de sujeto infinitiva, el verbo subordinado choqay tiene como objeto directo a la palabra castellana taba y por ello lleva la marca de acusativo - $t a$, y por el otro, que el verbo intransitivo gustay incrementa su valencia mediante el aplicativo - $p$ u para incorporar un objeto aplicado de $3 \cdot{ }^{\text {a }}$ persona que, externamente, estaría representado por el pronombre pay 'él'.
(pay-ta)
gusta-po-q
taba-ta
choqa-y-qa
él/ella-ACC gustar-APPL-PST.H taba-ACC arrojar-INF-TOP
'(a él) le gustaba el tirar la taba'

Por último, de manera similar a los aplicativos $-w a \sim-a$ y $-s u$ cuando ocurren con ciertos verbos intransitivos de sensación, como nanay 'doler' y chiriy 'hacer frío', el sufijo - $p u$ también incrementa la valencia verbal para traer a un participante periférico al centro del escenario convirtiéndolo en un objeto directo, de $3 .^{a}$ persona en este caso.

$\begin{array}{lll}\text { uma- } n & (\text { pay-ta }) & \text { nana-pu- } n \\ \text { cabeza-3POSS } & \text { él/ella-ACC } & \text { doler-APPL-3 }\end{array}$

'(a él) le duele su cabeza'

\section{Los aplicativos y el concepto de «tener»}

Entre las diversas construcciones posesivas en la quichua de Santiago del Estero, una muy recurrida es la que se forma mediante el verbo transitivo apiy 'agarrar', que se caracteriza porque lo poseído es algo externo al posesor, y en tal caso el verbo se traduce por 'tener'. Por ejemplo: 


$\begin{array}{lll}\text { kaqchi-situ-s-t } & \text { noqá } & \text { api-ni } \\ \text { perro-DIM-PL-ACC } & \text { yo-TOP } & \text { tener-I } \\ \text { 'yo tengo perros pequeños que no son de raza' }\end{array}$

Adicionalmente, en la quichua es posible generar estructuras posesivas a partir del verbo intransitivo tiyay 'estar, haber', mediante el uso de los aplicativos. Myler (2018, p. 157) ha realizado una exhaustiva descripción de esta construcción predicativa de posesión mediante tiyay pero acompañado únicamente del sufijo aplicativo - $p$ u y ofrece una detallada lista de las relaciones de posesión que se pueden expresar mediante la misma a partir de los datos obtenidos en su trabajo de campo. Myler (2018, p. 160) sostiene que, en general, los hablantes aceptan esta construcción «siempre que se pueda pensar que lo poseído está de alguna manera incrustado en, sobre, o que crece a partir del poseedor; por lo tanto, partes del cuerpo, partes de entes inanimados, parásitos, propiedades abstractas, estados mentales y enfermedades son todos aceptables».

Advertimos cierta discrepancia entre los registros obtenidos por Myler y nuestras propias observaciones en cuanto a las restricciones que esta construcción impone a lo poseído. Estas diferencias ameritan la apertura de una línea de investigación paralela sobre la cual estamos trabajando y oportunamente daremos a conocer los resultados alcanzados.

Por el momento, nos limitaremos a mostrar ciertos casos en los que los sufijos $-w a \sim-a$ y $-s u$, se comportan como aplicativos cuando se utilizan con el verbo intransitivo tiyay 'estar, haber', para formular relaciones de posesión aumentando la valencia a los efectos de incorporar un actante en función de objeto. Los siguientes son ejemplos para las $1 .^{\mathrm{a}}$ y $2 .^{\mathrm{a}}$ personas objeto, indexadas en la base verbal mediante $-w a \sim-a$ y $-s u$, respectivamente. Opcionalmente puede haber un pronombre correferente marcado con el acusativo -ta:

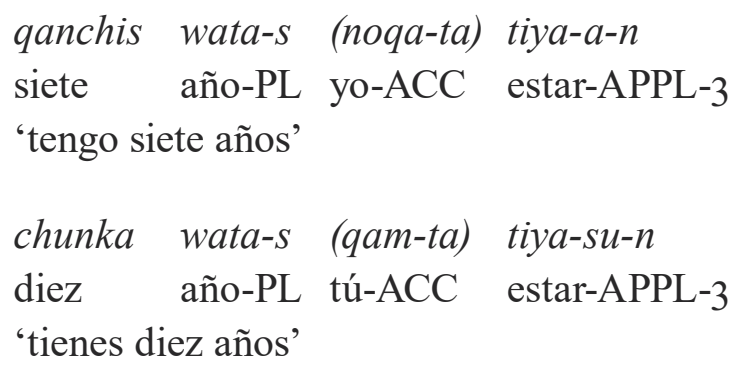


En cuanto al aplicativo - $p u$ en general hace referencia a la posesión de algo que corresponde a la $3 .{ }^{\text {a }}$ persona objeto, la cual está indexada en la base verbal mediante - pu. Si comparamos los ejemplos siguientes, notaremos que el sujeto de la oración intransitiva ('un defectito') se mantiene como tal en la estructura aplicativa y que - $p u$ incrementa la valencia verbal para poder incorporar a qari como objeto y con función semántica de Paciente.

suk-lla defectitu tiya- $n$
un-DLM defectito haber-3
'hay un solo defectito'

ka qari-ta tiya-pu-n suk-lla defectitu este hombre-ACC estar-APPL-3 un-DLM defectito 'este hombre tiene un solo defectito'

En el siguiente ejemplo, relacionado con la edad de la persona, el aplicativo se encuentra en el verbo subordinado:

$$
\begin{array}{ccccll}
\text { treinta } & \text { wata-S-manta } & \text { astaan-qa } & \text { mana } & \text { debe-ra- } & \text { tiya-pu-y-ta } \\
\text { treinta } & \text { año-PL-ABL } & \text { más-TOP } & \text { no } & \text { deber-PST-3 } & \text { estar-APPL- } \\
& & & & & \text { INF-ACC }
\end{array}
$$

'no debió haber tenido más de treinta años'

En estos otros ejemplos, la relación de posesión tiene que ver con las partes de un todo:

$\begin{array}{llllll}k a & \text { puchero-ta } & \text { tiya-pu-n } & \text { papa-s } & \text { sapallu } & \text { choklo-pas } \\ \text { este puchero-ACC } & \text { estar-APPL-3 } & \text { papa-PL } & \text { zapallo } & \text { choclo-TOO } \\ \text { 'este puchero tiene papas, zapallo y choclo' } & & \end{array}$
'este puchero tiene papas, zapallo y choclo'

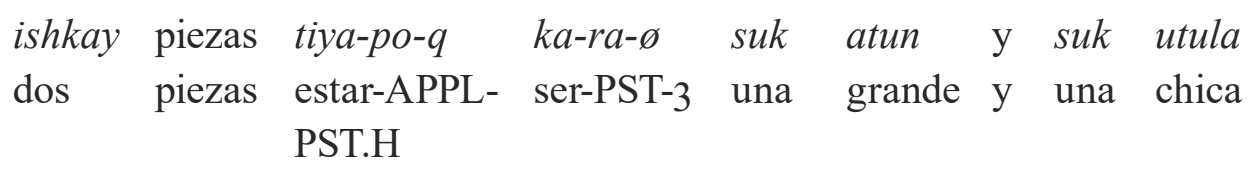

'Tenía dos piezas, una grande y una chica' 
Variación actancial en quichua santiagueño: los aplicativos (parte I)

Lelia Inés Albarracín y Regina Fernanda Gómez Nazar

$\begin{array}{llllll}\text { utula población } & \text { tiya-po-q-qa } & k a-r a-n k u & \text { Mariu-pa } & \text { ayllu-s-ni-n } \\ \text { poca población } & \text { estar-APPL- } & \text { ser-PST-3 } & \text { Mario-GEN } & \text { pariente-PL- } \\ & & \text { PST.H-TOP } & & & \text { EST-3POSS }\end{array}$

'la poca población que tenía eran parientes de Mario'

Es probable que en la quichua de Santiago del Estero también haya habido una estructura posesiva a partir del verbo kay 'ser' y el aplicativo - $p u$ a juzgar por algunos vestigios tales como la voz kapusqayki que aparece en una canción de mediados del siglo xix ${ }^{4}$; también la voz kapu que designa a 'un juego de niños consistente en apoderarse de lo que el otro tiene en la mano haciéndoselo tirar al suelo con un golpe' y, finalmente, la voz kapujay que significa 'arrebatar con agilidad y de manera súbita, atrapar una cosa que viene por el aire".

\section{Conclusiones}

En nuestra tarea docente advertimos la necesidad de implementar estrategias pedagógicas que permitan a los alumnos una mejor comprensión de los mecanismos de ajuste valencial y en particular, aquellos relacionados con los sufijos aplicativos. Además, entre nuestros colaboradores bilingües, hay cierto consenso en cuanto a que el verbo apiy en su significación de 'tener', está desplazando a las construcciones aplicativas que establecen relaciones de posesión, hecho verificable especialmente en las generaciones más jóvenes. Estas dos cuestiones forman parte de una serie de temas de nuestro interés, cuyo desarrollo consideramos necesario para la instrumentación de herramientas pedagógicas con destino a la formación de nuestros estudiantes de lingüística quichua. En este primer reporte de nuestra investigación sobre los mecanismos de ajuste de valencia mediante aplicativos, hemos presentado los resultados obtenidos para las estructuras provenientes únicamente de verbos intransitivos. En un próximo informe, mostraremos los resultados considerando verbos de diferente estructura valencial y describiremos casos de combinaciones entre aplicativos, sus restricciones y su posible interpretación.

4 «La Arunguita», según Grosso (2008, p. 121) es una chacarera de autor anónimo, de 1850 o 1860, aproximadamente.

28 Lengua y Sociedad. Revista de Lingüística Teórica y Aplicada 


\section{Referencias bibliográficas}

Albarracín de Alderetes, L. I. (2011). La Quichua Volumen II. Gramática, Ejercitaciones y Diccionario Quichua-Castellano. Dunken.

Albarracín de Alderetes, L. I. (2016). La Quichua Volumen III. Gramática, Ejercicios y Selección de Textos Quichuas. Dunken.

Albarracín de Alderetes, L. I. y Alderetes, J. R. (2013). El sufijo -pu en el quichua de Santiago del Estero. En M. Malvestitti y P. Dreidemie (Comps.), III Encuentro de Lenguas Indígenas Americanas. Libro de Actas (pp. 23-33). Universidad Nacional de Río Negro.

Albó, X. (1977). El quechua a su alcance (Vol. 1). Instituto de Cultura Indígena.

Alderetes, J. R. (2001). El Quichua de Santiago del Estero. Gramática y Vocabulario. Universidad Nacional de Tucumán, Facultad de Filosofía y Letras.

Carrió, C. (2013). Clasificación y análisis de las construcciones direccionales y locativas en mocoví. En M. Censabella y C. Messineo (Eds.), Lenguas indígenas de América del Sur II. Morfosintaxis y contacto de lenguas (pp. 39-57). FFyLUNCuyo y SAL.

Carrió, C. (2015). Alternancias Verbales en Mocoví (Familia Guaycurú, Argentina). Lingüística, 31(2), 9-26.

Censabella, M. (2011) Gramaticalización del aplicativo posicional-Rot en toba. En A. Fernández Garay y A. Díaz-Fernández (Eds.), Investigaciones sobre lenguas indígenas sudamericanas (pp. 41-68). Universidad Nacional de La Pampa.

Fernández Garay, A. (2012). Lingüística areal: las construcciones aplicativas en algunas lenguas patagónicas. Anuario de la Facultad de Ciencias Humanas, 1o(1), 5-13. https://cerac.unlpam.edu.ar/index.php/anuario/issue/view/68

González, R. E. (2016). Indexación de argumentos no-sujeto promovidos por aplicativos en Toba del Este de Formosa (Argentina). Forma y Función, 29(1), 39-61.

Grosso, J. L. (2008). Indios muertos, negros invisibles. Hegemonía, Identidad y Añoranza. Encuentro.

Itier, C. (1997). Parlons Quechua. La langue du Cuzco. París: L'Harmattan.

Marantz, A. (1981). On the nature of grammatical relations. Massachusetts Institute of Technology.

Martin, J. B. (2000). Creek Voice: Beyond Valency. En R. M. W. Dixon y A. Y. Aikhenvald (Eds.), Changing Valency (pp. 375-403). Cambridge University.

Myler N. (2016). Building and Interpreting Possession Sentences. Massachusetts Institute of Technology. 
Variación actancial en quichua santiagueño: los aplicativos (parte I)

Lelia Inés Albarracín y Regina Fernanda Gómez Nazar

Myler N. (2018). Variation in the Syntax and Semantics of Predicative Possession in Quechua. En M. Keough, N. Weber, A. Anghelescu, S. Chen, E. Guntly, K. Johnson, D. Reisinger y O. Tkachman (Eds.), Proceedings of the Workshop on the Structure and Constituency of the Languages of the Americas 21 (pp. 154-168). University of British Columbia.

Nardi, R. (2002). Introducción al quichua santiagueño. Dunken.

Polinsky, M. (2005). Applicative Constructions. En M. Haspelmath, M. S. Dryer, D. Gil y B. Comrie (Eds.), The World Atlas of Language Structures (pp. 442-445). Oxford University.

Payne, T. E. (2002 [1997]). Describing Morphosyntax. A Guide for Field Linguists. Cambridge University.

Peterson, D. A. (2007). Applicative Constructions. Oxford University.

Pylkkänen, L. (2008). Introducing Arguments. Massachusetts Institute of Technology.

Queixalós, F. y García Rivera, F. (2003). Efectos morfológicos de la pragmática en el verbo quechua. En G. Solís Fonseca (Ed.), Cuestiones de Lingüistica Amerindia. Tercer Congreso Nacional de Investigaciones Lingüísticas-Filológicas (pp. 105-119). PROEIB-Andes, GTZ, UNMSM y UNA. 


\section{Anexo}

Abreviaturas utilizadas:

\begin{tabular}{|c|c|c|}
\hline I & $-n i$ & I. ${ }^{\text {a }}$ persona verbal sujeto \\
\hline IOBJ & $-w a \sim-a$ & I. ${ }^{a}$ persona objeto \\
\hline IPOSS & $-y k i$ & I. ${ }^{a}$ persona posesora \\
\hline 2 & $-n k i$ & 2. ${ }^{\mathrm{a}}$ persona verbal sujeto \\
\hline 2IMP & $-y$ & 2. ${ }^{\mathrm{a}}$ persona imperativo directo \\
\hline 2OBJ & $-s u$ & 2. ${ }^{\mathrm{a}}$ persona objeto \\
\hline 2POSS & $-y k i$ & 2. ${ }^{\mathrm{a}}$ ersona posesora \\
\hline 3 & $-n /-n k u$ & 3. ${ }^{\mathrm{a}}$ persona verbal sujeto \\
\hline 3FUT & $-n q a$ & 3. ${ }^{\mathrm{a}}$ persona futuro \\
\hline 3OBJ & -pu & 3. ${ }^{\mathrm{a}}$ persona objeto \\
\hline 3POSS & $-n /-n k u n a$ & 3. ${ }^{\mathrm{a}}$ persona posesora \\
\hline 3ЕXH & -chun & 3. ${ }^{\mathrm{a}}$ persona exhortativo \\
\hline $\mathrm{ABL}$ & -manta & ablativo \\
\hline $\mathrm{ACC}$ & $-t a$ & acusativo \\
\hline ADV & $-t a$ & adverbializador \\
\hline APPL & $-p u /-w a /-s u$ & aplicativo \\
\hline AUX & & auxiliar \\
\hline BEN & $-p a q$ & benefactivo \\
\hline COND & - man & condicional \\
\hline DIM & -situ & diminutivo \\
\hline DLM & $-l l a$ & delimitador \\
\hline DS & $-p t i$ & distinto sujeto \\
\hline DUR & $-\operatorname{chka}$ & durativo \\
\hline EST & $-n i-$ & estribo \\
\hline EXH & & exhortativo \\
\hline GEN & $-p a$ & genitivo \\
\hline IMP & & imperativo \\
\hline INF & $-y$ & infinitivo \\
\hline LOC & $-p i$ & locativo \\
\hline MS & $-s p a \sim-s$ & mismo sujeto \\
\hline NEG & $-\operatorname{chu}$ & negativo \\
\hline NMLZ & $-n a$ & nominalizador \\
\hline OBJ & & objeto \\
\hline
\end{tabular}




\begin{tabular}{|c|c|c|}
\hline \multirow{2}{*}{\multicolumn{2}{|c|}{$\begin{array}{l}\text { OBL } \\
\text { OD }\end{array}$}} & oblicuo \\
\hline & & objeto directo \\
\hline PST & $-r a$ & pasado \\
\hline PL & & plural \\
\hline POSS & & posesivo \\
\hline PST.H & $-q$ & pasado habitual \\
\hline PST.N & $-s a$ & pasado narrativo \\
\hline $\mathrm{S}$ & & sujeto \\
\hline TOO & -pas & aditivo \\
\hline TOP & $-q a$ & topicalizador \\
\hline
\end{tabular}

\section{Trayectoria académica de los autores}

\section{Lelia Inés Albarracín}

Profesora Adjunta responsable de los espacios curriculares Lingüística Quichua y Taller de Lengua Quichua en la Carrera Tecnicatura Superior en EIB de la Facultad de Humanidades, Ciencias Sociales y de la Salud de la Universidad Nacional de Santiago del Estero. En la misma unidad académica tiene a su cargo el dictado de las asignaturas sobre lingüística quichua de la Diplomatura en Lengua Quichua.

\section{Regina Fernanda Gómez Nazar}

Técnico Superior Universitario en Educación Intercultural Bilingüe con Mención en Lengua Quichua por la Universidad Nacional de Santiago del Estero. Participa en proyectos de investigación en la citada universidad y es miembro de la Asociación de Investigadores en Lengua Quechua (ADILQ). 